\title{
Impact of temporal variation and the balance between excitation and inhibition on the output of the perfect integrate-and-fire model
}

\author{
Jianfeng Feng, David Brown \\ Biomathematics Laboratory, The Babraham Institute, Cambridge CB2 4AT, UK \\ Received: 25 August 1997 / Accepted in revised form: 19 February 1998
}

\begin{abstract}
We consider how the output of the perfect integrate-and-fire (I\&F) model of a single neuron is affected by the properties of the input, first of all by the distribution of afferent excitatory and inhibitory postsynaptic potential (EPSP, IPSP) inter-arrival times, discriminating particularly between short- and longtailed forms, and by the degree of balance between excitation and inhibition (as measured by the ratio, $r$, between the numbers of inhibitory and excitatory inputs). We find that the coefficient of variation $(\mathrm{CV}$; standard deviation divided by mean) of efferent interspike interval (ISI) is an increasing function of the length of the tail of the distribution of EPSP inter-arrival times and the ratio $r$. There is a range of values of $r$ in which the CV of output ISIs is between 0.5 and 1 . Too tight a balance between EPSPs and IPSPs will cause the model to produce a $\mathrm{CV}$ outside the interval considered to correspond to the physiological range. Going to the extreme, an exact balance between EPSPs and IPSPs as considered in [24] ensures a long-tailed ISI output distribution for which the moments such as mean and variance cannot be defined. In this case it is meaningless to consider quantities like output jitter, $\mathrm{CV}$, etc. of the efferent ISIs. The longer the tail of the input inter-arrival time distribution, the less is the requirement for balance between EPSPs and IPSPs in order to evoke output spike trains with a CV between 0.5 and 1 . For a given short-tailed input distribution, the range of values of $r$ in which the CV of efferent ISIs is between 0.5 and 1 is almost completely inside the range in which output jitter (standard deviation of efferent ISI) is greater than input jitter. Only when the CV is smaller than 0.5 or the input distribution is a long-tailed one is output less than input jitter [21]. The I\&F model tends to enlarge low input jitter and reduce high input jitter. We also provide a novel theoretical framework, based upon extreme value theory in statistics, for estimating output jitter, $\mathrm{CV}$ and mean firing time.
\end{abstract}

Correspondence to: J. Feng

\section{Introduction}

The integrate and fire (I\&F) model is a rudimentary model that underpins the idea of a neuron being a device which accumulates inputs until it reaches a threshold, whereupon it fires. The model has recently been examined by several authors [1, 24, 25, 28]. Softky and Koch [25] compared the behaviour of the model with data obtained in the visual cortex of the monkey, via theoretical calculations and numerical simulations. They found, with exclusively excitatory postsynaptic potential (EPSP) inputs, that the output of the I\&F model is very regular, with the coefficient of variation (CV) of the inter-spike interval (ISI, the standard deviation or so-called output jitter divided by the mean) converging to zero at a rate $1 / \sqrt{N_{\text {th }}}$, as $N_{\text {th }}$ becomes large, where $N_{\text {th }}$ is the number of EPSPs needed to trigger a spike. Therefore, they concluded that the model cannot account for the phenomena observed in neurones in the visual cortex with a $\mathrm{CV}$ between 0.5 and 1 . Their results cast doubt on the rate coding assumption of neural computation. In 1994, Shadlen and Newsome [24] reconsidered the same model and claimed that an equal balance between EPSPs and inhibitory PSPs (IPSPs) can get around this difference between model prediction and experimental data, i.e. that the model is capable of producing sufficiently variable output. Due to the biologically unprovable assumption about the exact balance between EPSPs and IPSPs in the random walk model treated in [24], Troyer and Miller [28] turned their attention to modifying the mechanism and showed, mainly by numerical simulations, that the model exhibits physiologically reasonable behaviour, i.e. output $\mathrm{CV}$ is between 0.5 and 1 , if the after-spike reset voltage is higher than the resting potential. ${ }^{1}$

\footnotetext{
${ }^{1}$ We concentrate on experimental data reported in [25] for the visual system of the monkey, to be consistent with parameters used in our numerical simulations later on. There are, however, many reports of experimental results with a $\mathrm{CV}$ outside this region (see for example $[15,16])$.
} 
In another closely related paper, Marsalek, Koch and Maunsell [21] showed that output jitter in the I\&F model converges to zero as the number of inputs becomes large if inter-arrival time distributions of EPSPs are normally, uniformly or exponentially distributed. Based upon further extensive numerical simulations on more realistic neuronal models, they then argued that this property of neurones provides one of the biophysical substrates necessary for exploiting the detailed timing information inherent in spike trains $[2,9,10]$.

However, there are several fundamental issues which have not been fully taken into account in the previous discussion on the perfect $\mathrm{I} \& \mathrm{~F}$ model (see also $[15,29])$.

The discussion is restricted to the case in which the distribution of inter-arrival times of EPSPs or IPSPs are short-tailed, i.e. the tail goes to zero exponentially, the negative exponential and Gaussian distributions being special cases. This is probably one of the most important models in neural computation [1]. However, as early as 1964, Gerstein and Mandelbrot [12] pointed out that the output distributions of neurones could also be longtailed, where the tail of a distribution tends to zero geometrically, the Pareto distribution being a special case. Additionally, the output of the I\&F model can be either long- or short-tailed, as shown in Section 3. A further important consideration is that after the nonlinear, complex manipulation of dendritic trees [19], it is not at present clear what the distributions of EPSPs and IPSPs are when they reach the point at which integration occurs - supposed to be the axon hillock - even assuming that inputs from all distant and proximal synapses are themselves Poisson processes. Therefore, it is safer and probably more illuminating to consider the behaviour of quantities like output jitter, CV, etc. under different assumptions about the distributions of interarrival times of EPSPs and IPSPs. In summary, the issue is to consider how the output of a neuron is affected by input variation in the time domain.

The phenomena revealed in the above-mentioned literature are usually based upon results for specifically chosen values of each parameter. It seems worthwhile carrying out a complete, thorough investigation for wide ranges of a few physiologically important parameters. Because of the lack of accurate information on $r$, the ratio between inhibitory and excitatory inputs (see $[2,4]$, p. 53 for a discussion about estimation of $r$ ), it is reasonable to consider a wide range of possible values of $r$, reflecting different degrees of balance between inhibitory and excitatory inputs.

It is critical to consider characteristics of the output like output jitter, mean firing time and CV together in a systematic manner rather than separately, as some previous studies have done. In considering the feasibility of a model, all these responses must be in the physiological range. This paper demonstrates that models are assessed differently when all three aspects of the output are taken into account.

Bearing the above three issues in mind, we thus consider the model with input distributions of different tail lengths, and spanning a wide range of values of $r$. Some basic questions addressed are the following:

1. Is output jitter smaller than input jitter in the parameter region in which the model also produces a physiologically reasonable $\mathrm{CV}$, i.e. between 0.5 and 1? How does the behaviour of the output jitter depend on the nature (i.e. long- or short-tailed) of the input distributions?

2. Are equal excitatory and inhibitory inputs needed to produce a physiologically reasonable CV? In other words, how large is the range of values of $r$, the ratio between inhibitory and excitatory inputs, in which the $\mathrm{CV}$ of the model is between 0.5 and 1 ? How does the range depend on the input distribution?

3. For a given input distribution, what is the corresponding output distribution?

We are able to answer questions 1 and 2 completely and question 3 partially, providing considerable insight about the relationship between input and output of the I\&F model. To deal theoretically with long-tailed distributions is usually intractable since the Central Limit Theorem in the usually quoted form is not applicable. In Sect. 4 we develop a novel theoretical framework to show how to estimate output jitter, mean firing time and $\mathrm{CV}$. In a simplified version of the I\&F model, we have considered the effect of input variation in the time domain, and, in that case, exact formulas for the efferent distributions of ISIs are obtained $[9,10]$.

\section{The model}

Basically, the I\&F model $[13,18,29]$ is a threshold device charged with sequences of EPSPs and IPSPs. The model consists of $N_{E}$ asymmetric synapses (excitatory inputs) and $N_{I}$ symmetric synapses (inhibitory inputs). Each excitatory and inhibitory synapse dumps a sequence of EPSPs and IPSPs, respectively, at the cell body. More specifically, let $T_{i}^{E, j}\left(T_{i}^{I, j}\right)$ be the inter-arrival time of the $i$ th EPSP (IPSP) which travels along the $j$ th excitatory (inhibitory) synapse. Then, $T_{i}^{E, j}, i=1,2, \ldots$, $j=1, \ldots, N_{E}$, are independently identically distributed random times, and so are $T_{i}^{I, j}, i=1,2, \ldots, j=1, \ldots, N_{I}$. Hence the arrivals of EPSPs or IPSPs at each synapse behave as a renewal process. Denote $V(t)$ as the membrane potential at time $t$. As soon as one EPSP arrives, say the $i$ th EPSP from the $j$ th synapse, the potential is depolarized from $V(t-)$ to $V(t-)+a$; the IPSP has the reverse effect, i.e. it hyperpolarizes the membrane potential by an amount $b$. Once $V(t)$ reaches the threshold $V_{\text {thre }}$, an output spike is generated and the membrane potential is reset to $V_{\text {rest }}$, the rest potential. More specifically, we have

$$
\frac{d V(t)}{d t}=a \sum_{j=1}^{N_{E}} \delta\left(t-\sum_{i=1}^{\infty} T_{i}^{E, j}\right)-b \sum_{j=1}^{N_{I}} \delta\left(t-\sum_{i=1}^{\infty} T_{i}^{I, j}\right)
$$


if $V(t)<V_{\text {thre }}$. For simplicity of notation, we suppose that a single IPSP has the same magnitude as a single EPSP, ${ }^{2} a=b=0.5 \mathrm{mV}$. Therefore, the threshold number of EPSPs ensuring the model evokes a spike is $N_{\text {th }}=40=V_{\text {thre }} / a$.

Let $T$ be the first time that $V(t)$ reaches threshold, i.e. the time for the membrane to produce a spike commencing from its resting potential. It is given by

$T=\inf \left\{t: V(t) \geq V_{\text {thre }} \mid V(0)=V_{\text {rest }}\right\}$

which represents the inter-spike interval of the output. In this paper we focus on the behaviour of $T$ under the following three input distributions:

(Positive) Gaussian input. $T_{i}^{E, j}$ is distributed as

$$
\frac{2}{\sqrt{2 \pi}} \exp \left(-\frac{t^{2}}{2}\right) I_{\{t \geq 0\}}
$$

where $I_{A}(\cdot)$ is the indicator function for a set $A$, i.e. $I_{A}(t)=1$ if $t \in A$ and 0 otherwise.

Poisson input. $T_{i}^{E, j}$ is an exponentially distributed random variable, i.e. its density is

$\exp (-t) I_{\{t \geq 0\}}$

Pareto input. The distribution density of $T_{i}^{E, j}$ is

$$
\alpha(t+1)^{-\alpha-1} I_{\{t \geq 0\}}
$$

with $\alpha>0$. In this paper we take $\alpha=1$ and $\alpha=2.1$ exclusively, and thus we have $E T_{1}^{E, 1}=\infty, E\left(T_{1}^{E, 1}\right)^{2}=$ $\infty$ for $\alpha=1$ and $E T_{1}^{E, 1}=0.91, \sigma_{\text {in }}=4.16$ for $\alpha=2.1$. The longer the tail, the larger the temporal variation of inputs is.

In the following when we speak of Gaussian, Poisson and Pareto distributed inputs, we mean $T_{1}^{E, 1}$ is distributed as (1)-(3), i.e. the inter-arrival times of the EPSPs and IPSPs at each synapse are distributed according to (1)-(3) respectively. The Gaussian and the exponential distributions are short-tailed distributions, while the Pareto distribution has a long tail. We can write them in a unified form $c \exp (-f(t))$ for a constant $c$, where $f(t)$ is $t^{3} / 2$ for the Gaussian distribution, $t$ for the exponential distribution and $(\alpha+1) \log (t+1)$ for the Pareto distribution. Hence, the Pareto distribution has the longest tail, going slowly to zero at a rate of $\exp (-\log t)$, whereas the tail of the exponential distribution tends to zero at a rate of $\exp (-t)$, more slowly than that of the Gaussian distribution, which approaches zero at a rate of $\exp \left(-t^{2}\right)$. It should be emphasized here that there is an essential difference between the Poisson and Gaussian or Pareto inputs. For Poisson inputs, the EPSP and IPSP processes can start from zero again after a spike, as the Poisson process has no memory. For Gaussian or Pareto inputs, we do not have this property.

\footnotetext{
${ }^{2}$ For an estimation of activity-dependent magnitude of EPSPs, see [27].
}

The mean firing time $E T$ is the expectation of $T$, and output jitter is defined by

$\sigma_{\text {out }}=\sqrt{E T^{2}-(E T)^{2}}$

provided it makes sense, i.e. $E T^{2}<\infty$, and $C V$ is given by

$C V=\sigma_{\text {out }} / E T$

under the condition that $0<E T<\infty$. The mean and standard deviation of the exponential distribution (2) are both unity. All properties of the model in this case are scale-independent, so our discussion of this case also includes the more general case in which the mean and standard deviation are not unity. The occurrences of contractive or expanding mappings, denoted by $\sigma_{\text {out }} / \sigma_{\text {in }}>1$ and $\sigma_{\text {out }} / \sigma_{\text {in }}<1$, corresponds for these parameters to $\sigma_{\text {out }}>1$ and $\sigma_{\text {out }}<1$, respectively.

\section{Results}

\subsection{Output jitter}

We first consider output jitter, which is determined solely by the parameters of the model. It is known [7, 29] that output jitter for Poisson input is (see Fig. 1)

$\sigma_{\text {out }}=\sqrt{\frac{1+r}{(1-r)^{3}}} \cdot \frac{\sqrt{q}}{\sqrt{N_{E}}}$

where $r=N_{I} / N_{E}$, the ratio between the numbers of inhibitory and excitatory synapses, $q=N_{\text {th }} / N_{E}$, and $r<1$. In Fig. $1 \mathrm{~b}$ we numerically calculate the output jitter at $r=0.1,0.2,0.3, \ldots, 0.9$ for Pareto and Gaussian inputs with $N_{E}=100$, which is about $3 \%-4 \%$ of the number of excitatory input synapses of a pyramidal cell in the visual cortex [24]. The output jitter of the Pareto inputs is fitted, for $r<1$, by [see next section for explanation (14)]

$\sqrt{\frac{1+r}{(1-r)^{4.6}}} \cdot \sqrt{\frac{q}{N_{E}}}$

From (6), (7) and a study of Fig. 1, we can make the following observations.

The better the balance between inhibitory and excitatory inputs ( $r$ getting closer to 1$)$, the larger the output jitter is. For Poisson inputs (Fig. 1a), when $r>0.8$, we see that $\sigma_{\text {out }}>1$. This implies that under the condition $r>0.8$, the processing of the model cell forms an expanding mapping, i.e. $\sigma_{\text {out }} / \sigma_{\text {in }}>1$ rather than a contractive one, i.e. $\sigma_{\text {out }} / \sigma_{\text {in }}<1$. Only when $r<0.8$ does the cell impose a contractive mapping. In other words, results described in [21] are true only when $r<0.8$. Figure $1 \mathrm{~b}$ shows the output jitter with the Pareto distribution inputs with $\alpha=1$ : when $r>0.6$, the output jitter is greater than 1. For a given afferent distribution the output jitter is therefore an increasing function of the ratio between inhibitory and excitatory inputs. As soon as an equal balance between inhibitory and excitatory inputs is reached, which means $N_{I}=N_{E}$, the case con- 

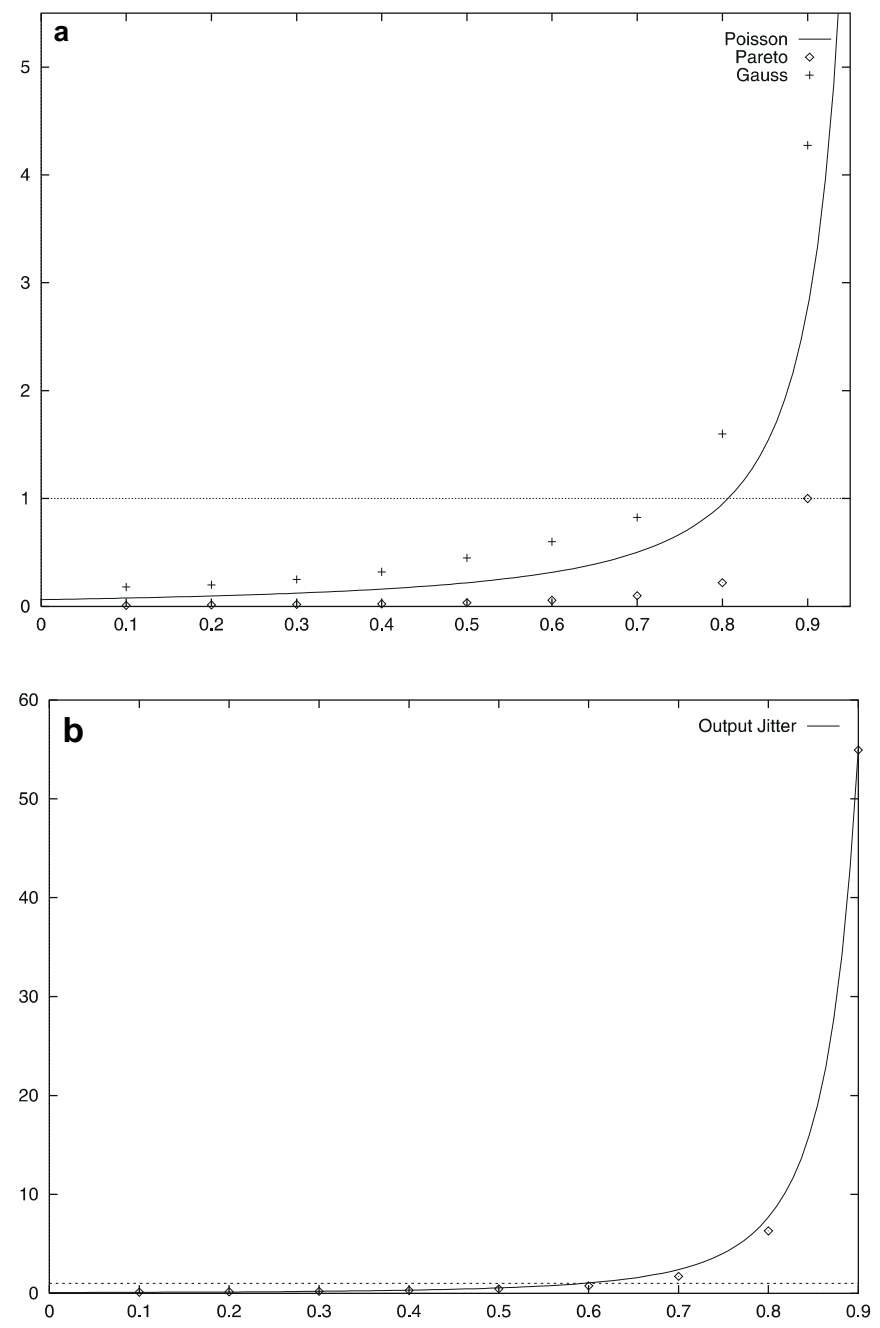

Fig. 1 a Output jitter/input jitter vs the ratio between the numbers of inhibitory and excitatory inputs. The solid line is the output jitter/ input jitter of the Poisson input. $\diamond$ and + are obtained via numerical simulations for the Gaussian and Pareto $(\alpha=2.1)$ distributed input. When $r$ is about 0.8, the output jitter is larger than 1 for the Poisson input. However, for the Pareto input with $\alpha=2.1$ we see that the output jitter is always smaller than the input jitter when $r \leq 0.9$. b Output jitter vs the ratio between the numbers of inhibitory and excitatory inputs for the Pareto distributed input with $\alpha=1$. $\diamond$ is obtained via numerical simulations, and the data are fitted by (7) (solid line). When $r$ is about 0.6 , the output jitter is 1 for the Pareto input

sidered in [24], output jitter is infinity for both the Poisson (6) and Pareto inputs (7). Note that the output jitter for the Poisson input is a multiplication of two terms: first $\sqrt{N_{\text {th }}} / N_{E}=\sqrt{q} / \sqrt{N_{E}}$ which is related to the ratio $q$ and tends to zero as $\sqrt{N_{E}}$, a phenomena similar to that of the Central Limit Theorem which has been pointed out by several authors $[21,24,28]$ for short-tail inputs; the second term is $f(r)=\sqrt{1-r} / \sqrt{(1-r)^{3}}$ which reflects the influence of the ratio between inhibitory and excitatory synapses.

Output jitter is also an increasing function of the weight in the tail of the input distribution. The fatter the tail, the larger the output jitter becomes. For example, when $r=0.6$, the output jitters of the Gaussian input and Poisson input are less than 0.5, whereas for the Pareto input with $\alpha=1$, the output jitter is about 1 .

\section{$3.2 \mathrm{CV}$ and mean firing time}

In this subsection we answer the first and the second questions raised in Sect. 1 completely.

1.The $C V$ for Poisson input is $[7,29]$

$$
C V=\sqrt{\frac{1+r}{N_{\mathrm{th}}(1-r)}}=\sqrt{\frac{1+r}{1-r}} \cdot \frac{1}{\sqrt{q N_{E}}}
$$

and the mean firing time is

$$
E T=\frac{N_{\mathrm{th}}}{N_{E}} \cdot \frac{1}{1-r}=\frac{1}{1-r} \cdot q
$$

Before proceeding, let us introduce a notation to facilitate our further discussion. For a given input distribution define $C V$ as a function of $r$ denoting $C V(r)$, the ratio between inhibitory and excitatory inputs.

$r_{0.5}=\inf \{r: C V(r)>0.5\}$

the threshold of the ratio at which the $C V$ is 0.5 and

$r_{1}=\sup \{r: C V(r)<1\}$

the threshold of the ratio at which the $C V$ is 1 . For a given input distribution $F$, the following quantity

$\mathscr{M}(F):=\frac{1}{r_{1}-r_{0.5}} \int_{r_{0.5}}^{r_{1}} \frac{\sigma_{\text {out }}}{\sigma_{\text {in }}} d r$

characterizes the relationship between input jitter and output jitter.

Definition 1 For a given input distribution $F$, the $I \& F$ model is a contractive mapping of the jitter if $\mathscr{M}(F)<1$ and an expanding mapping of the jitter if $\mathscr{M}(F)>1$

In Fig. 2 the $C V$ of differently distributed inputs is plotted vs $r$, the ratio between inhibitory and excitatory inputs, leading to the following conclusions.

1. For all distributions considered in this paper, $C V$ is an increasing function of the ratio between inhibitory and excitatory inputs, although we find both mean firing time (see Fig. 3) and output jitter (see Fig. 1) are also increasing functions of the ratio.

2. There is a range of the values of the ratio, $r$, in which a physiological $C V$ can be produced. It is not necessary to have an equal balance between excitatory and inhibitory inputs as required in [24]. In fact when too tight a balance between IPSPs and EPSPs occurs, the $C V$ is too high, i.e. greater than 1 , in comparison with cells in the visual cortex (see Fig. 2b: when $r=0.9$ the $C V$ is 3 ).

3. We see that the thresholds $r_{0.5}$ and $r_{1}$ are decreasing functions of the weight in the tail. In other words, the fatter the tail, the smaller the threshold is, which implies that less balance is required for producing a 

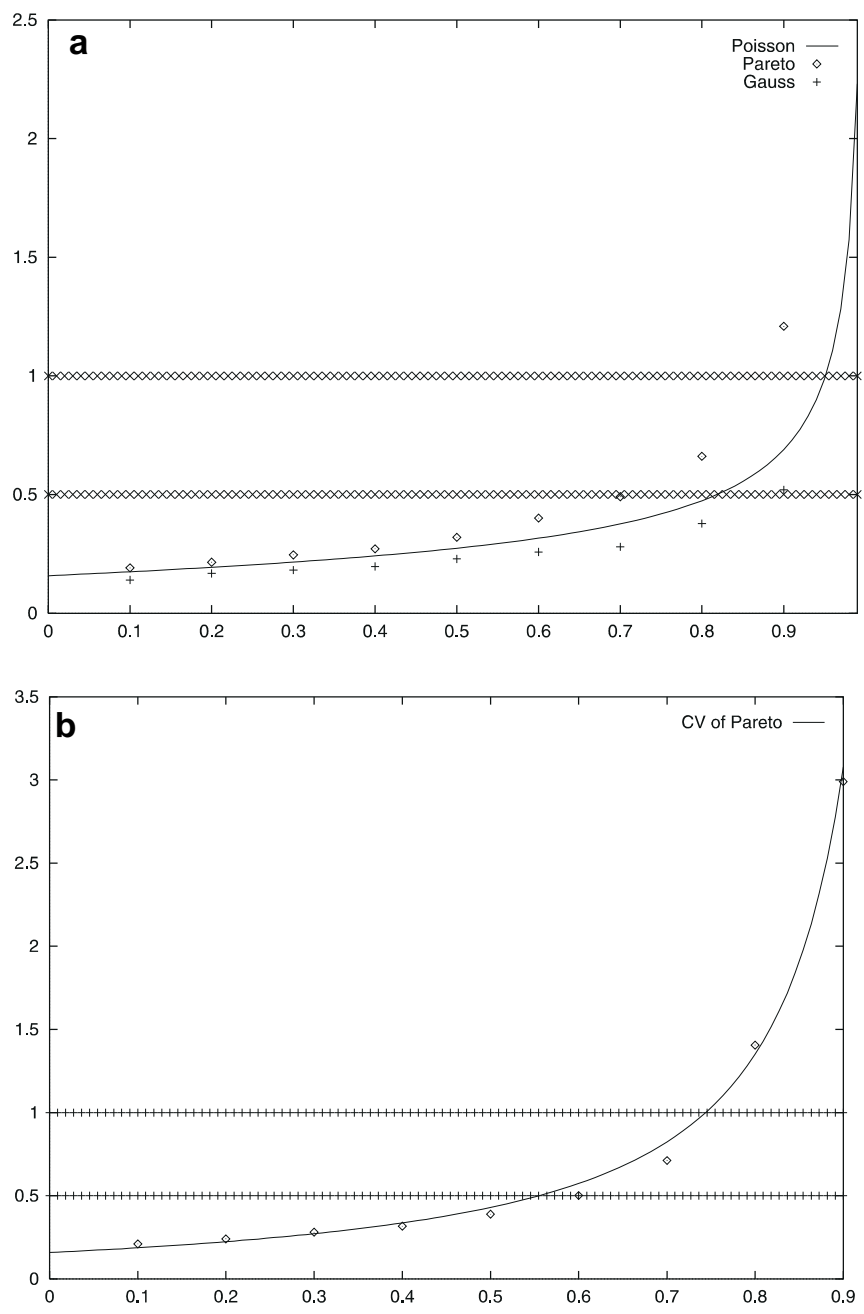

Fig. 2a,b. Coefficient of variation $(C V)$ vs the ratio between inhibitory inputs and excitatory inputs. As in Fig. 1, we numerically calculate the $C V$ at $r=0.1,0.2,0.3, \ldots, 0.9$ with $N_{E}=100$. There is a parameter region of $r$ in which the $C V$ is between 0.5 and 1: for the Pareto input with $\alpha=2.1$, we have $r_{0.5}=0.7, r_{1} \sim 0.89$; for the Gaussian input we have $r_{0.5}=0.9$. When the balance between inhibitory and excitatory inputs is too tight, the $C V$ is greater than 1. a $C V$ of the Gaussian, Poisson and Pareto inputs with $\alpha=2.1$. b $C V$ of the Pareto inputs with $\alpha=1$, the curve (solid line) ' $C V$ of Pareto' being $\sqrt{(1+r) /(1-r)^{2.3}} \cdot \sqrt{1 /\left(q N_{E}\right)}$

physiologically reasonable $C V$ in the case of input distributions with long tails.

4. For an input short-tailed distribution (Gaussian or Poisson) the threshold of $r$ at which $C V>0.5$ is inside the range of $r$ where output jitter/input jitter is greater than 1 (see Fig. 1). This indicates that as long as $r$ is within a region such that output $C V$ is within the physiological range (between 0.5 and 1), output jitter will be larger than input jitter. Output jitter is less than input jitter only when $C V$ is less than 0.5 . In [21] the authors observed that output jitter is lower than input jitter, and neurones can therefore more easily fire together in deeper layers of the brain. However, our results simply indicate that, as soon as the ratio is in the region which produces a physiological $C V$, output jitter will augment rather

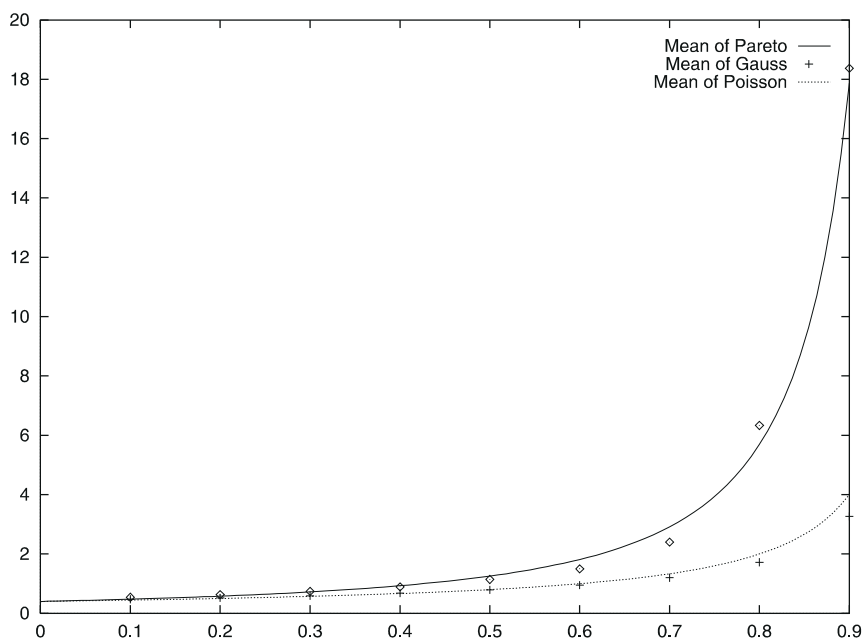

Fig. 3. Mean firing time of three different inputs vs $r$, the ratio between numbers of inhibitory and excitatory inputs. As before, we numerically calculate $E T$ at $r=0.1,0.2,0.3, \ldots, 0.9$ with $N_{E}=100$. + and $\diamond$ are obtained via numerical simulations of the Gaussian distribution and Pareto distribution with $\alpha=1$ and $N_{E}=100$. The curve 'Mean of Pareto' is $q /(1-r)^{1.65}$

than reduce input jitter (in the case of Poisson and Gaussian inputs).

5. If there is an exact balance between excitatory and inhibitory inputs as considered in [24], the output $C V$ will be infinite.

6. When the input is Poisson, the $C V$, output jitter and mean firing time are all composed of two terms. One reflects the effect of the ratio between EPSPs and IPSPs, while the other is related to the threshold number and total number of excitatory input synapses.

7. Synaptic inhibitory inputs help the neuron to adjust its firing in a significantly different manner. For fixed numbers of excitatory inputs, when the number of inhibitory inputs is high, the neuron will elicit spikes slowly (with high mean ISI) and irregularly (with high output jitter); whereas if the number of inhibitory inputs is low, the neuron will fire faster (lower mean ISI) and more regularly (lower output jitter). It is well known that a neuron subjected to changed synaptic inputs frequently evokes different output spike trains. There is also a large variety of neuronal behaviour, from chattering cells to neurones firing slowly and regularly, from individual phasic activity to neurones grouping together to oscillate. Obviously, the I\&F model cannot fully account for these phenomena, but it provides an insight, as revealed in this paper, on how synaptic inputs possibly influence the output.

8. We note that $\mathscr{M}($ Gauss $)>1, \mathscr{M}$ (Poisson) $>1$ and $\mathscr{M}($ Pareto, $\alpha=2.1)<1$. Hence, there are parameters $\alpha_{0}$ satisfying $\mathscr{M}\left(\right.$ Pareto, $\left.\alpha_{0}\right)=1$ (see Sect. 3). In other words, there are Pareto distributions which result in neither expanding nor contractive mappings.

9. Finally, we point out that (8) explains why a higher $C V$ is produced if the after-spike reset voltage is 
higher [28], since $C V$ is a decreasing function of $N_{\text {th }}$. For further discussion on the effect of partial reset or incomplete repolarization, see $[6,17]$.

\subsection{Output distributions}

As pointed out in the introduction, it is an intriguing problem to consider the relationship between input and output in terms of distributions. Although it is always possible to calculate the $C V$ and output jitter numerically, we run the risk that the numerical results may be meaningless. The output jitter and mean firing time may not exist as in the case of Pareto distribution (for $\alpha \leq 2$ ), and therefore in different realizations of the random dynamics of the I\&F model, we frequently obtain very different values of $C V$ and the output jitter. Unfortunately, obtaining, an analytical expression for the output distribution is difficult, and not always possible. The only analytical result we know is for Poisson-distributed inputs, which forms a typical birth-and-death process [8]. The distribution density is

$N_{\text {th }}\left(\frac{N_{E}}{N_{I}}\right)^{N_{t h} / 2} \frac{\mathrm{e}^{-\left(N_{E}+N_{I}\right) t}}{t} I_{N_{\mathrm{th}}}\left(2 t \sqrt{N_{E} N_{I}}\right)$

where $I_{N_{\mathrm{th}}}$ is the modified Bessel function (see [7, 29]). In particular, when $t$ is large, (10) turns out to be (see [29], p.134)

$\frac{\exp \left(-\left(\sqrt{N_{E}}-\sqrt{N_{I}}\right)^{2} t\right)}{t^{3 / 2}} \cdot$ constant

This formula tells us that as soon as an exact balance between EPSPs and IPSPs is reached, the output distribution is a long-tail distribution with tail $t^{-3 / 2}$. The mean firing time and output jitter of ISIs for the case considered in [24] do not exist.

Figure 4 tells us that the weight in the tail of the output distribution is an increasing function of both $r$, the ratio between inhibitory and excitatory synapses, and the tail length of the input distribution. For Poisson input we know that if and only if an exact balance between EPSPs and IPSPs is reached will the output distribution have a long tail. For the Pareto distribution, we see from Fig. $4 b$ that the tail of the output distribution becomes fatter as $r$ increases. However, it is not easy for us to tell, from numerical results only, whether the output distribution has a long tail or not (see Sect. 2). Here analytical approaches are crucial if we are ever to make sense of the data.

\section{A theoretical framework for estimating $\mathrm{CV}$, output jitter and mean firing time}

As we pointed out in the previous section, to the best of our knowledge it is not easy to find an analytical expression for the probability density of $T$ and, as a consequence, an analytical expression for output jitter and ISI $C V$. The difficulty lies in the fact that for
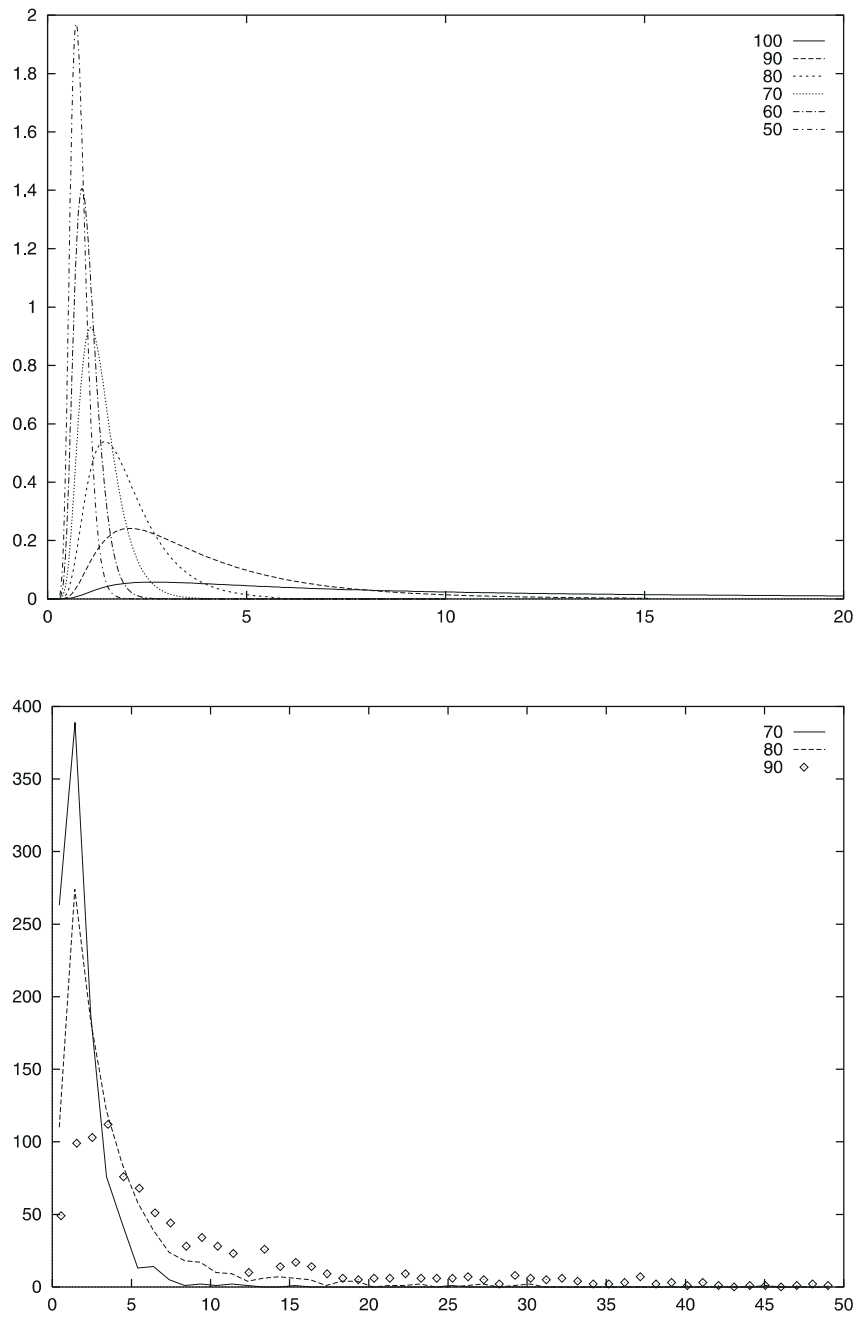

Fig. 4a,b. Distribution density of $T$, i.e. histogram of ISIs with $N_{E}=100$. Numbers shown on the right upper corner are $N_{I}$. a is obtained analytically. Note that as soon as $N_{I}=100=N_{E}$ the output probability density is a long-tail distribution (solid line). b Numerical results of ISIs when input is the Pareto distribution. It is obvious that the tail becomes fatter as $r$ increases

Gaussian and Pareto inputs the following property is lacking: the exponential distribution is linear in power, which enables us to carry out the Laplace transformation, and the superposition of Poisson processes is also a Poisson process [7, 29]. From the results presented in the previous section, we see that the $C V$, output jitter and mean firing time of Poisson inputs are all products of two factors: one is related to the ratio $r$, the other to $N_{E}, q$. In this section we provide an argument based upon extreme value theory to explain why there is a factor $1 / \sqrt{N_{E}}$ in the expression for $C V$ and output jitter, this factor being similar to that given by the Central Limit Theorem.

Let us first observe the relationship between the extreme values of $T_{1}^{E, 1}, \ldots, T_{1}^{E, N_{E}}$, the sequence of the first arrival times of the EPSPs of excitatory synapses, and the firing time $T$. Without loss of generality suppose that $T_{1}^{E, 1} \leq T_{1}^{E, 2} \leq \cdots \leq T_{1}^{E, N_{E}}$ 
In general for each random realization of the model, we expect that the neuron will fire before all synapses evoke an EPSP, which means that there exists $k, 1<k<N_{E}$ such that

$T_{1}^{E, k} \leq T \leq T_{1}^{E, k+1}$

Under the condition that $T_{1}^{E, k+1}-T_{1}^{E, k}$ is small (see the following lemmas), we have $T \sim T_{1}^{E, k}$. Denote $p=k / N_{E}$ for a constant $0<p<1$. Let $F(x)$ be the distribution function of $T_{1}^{E, 1}$. The following two lemmas can be proved.

Lemma 1 ([4,5]) Suppose that $F$ is differentiable at $y_{0}$ and $F\left(y_{0}\right)>0$. Set $p=F\left(y_{0}\right)$. Then $T_{1}^{E,\left[p N_{E}\right]}$ is asymptotically $\left(N_{E}\right.$ goes to infinity) normal $N\left(\mu_{N_{E}}, \sigma_{N_{E}}\right)$ with

$$
\left\{\begin{array}{l}
\mu_{N_{E}}=y_{0}=F^{-1}(p) \\
\sigma_{N_{E}}=\frac{\sqrt{p(1-p)}}{\sqrt{N_{E}} F^{\prime}\left(y_{0}\right)}
\end{array}\right.
$$

where [.] denotes the integer part of a real number.

The results of Lemma 1 are quite remarkable. It is easy to see the Pareto distribution has infinite variance for some parameter $\alpha>0$, and thus the Central Limit Theorem in the form usually quoted is not applicable. Lemma 1 is more powerful than the usual Central Limit Theorem: it only requires the distribution of $T_{1}^{E, 1}$ to be locally differentiable and is applicable to a wide range of distribution without the existence of mean and variance.

Lemma 2 For $0<p<1$ it we have the following conclusions in Table 1

We omit the proof of Lemma 2 here and only want to emphasize that there is a vanishing term $1 / \sqrt{N_{E}}$ for the standard deviation $S\left(T_{1}^{E,\left[p N_{E}\right]}\right)$ of $T_{1}^{E,\left[p N_{E}\right]}$ for all input distributions. Lemma 2 together with the above considerations tells us that

$$
\begin{aligned}
E T & =c_{1}(p) \cdot f_{1}(r) \\
\sigma_{\text {out }} & =\frac{c_{2}(p)}{\sqrt{N_{E}}} \cdot f_{2}(r)
\end{aligned}
$$

where $c_{1}(p), c_{2}(p)$ are functions related to the EPSP and IPSP inter-arrival time distribution, and $f_{1}(r), f_{2}(r)$ are related to the degree of balance between excitation and inhibition. For the Poisson distribution we have $c_{1}(p)=q, f_{1}(r)=1 /(1-r)$ and

Table 1. $\operatorname{Erf}(x)=\frac{2}{\sqrt{2 \pi}} \int_{0}^{x} \exp \left(-y^{2} / 2\right) d y$

\begin{tabular}{lll}
\hline Distribution & $\begin{array}{l}S\left(T_{1}^{E,\left[p N_{E}\right]}\right) \\
(\text { standard deviation) }\end{array}$ & $\begin{array}{l}E T_{1}^{E,\left[p N_{E}\right]} \\
(\text { mean })\end{array}$ \\
\hline Gaussian & $p^{1 / 2}(1-p)^{1 / 2} \exp \left(y_{0}^{2} / 2\right) /\left(\sqrt{2 \pi N_{E}}\right)$ & $y_{0}=\operatorname{Erf}^{-1}(p)$ \\
Pareto & $p^{1 / 2}(1-p)^{-1 / 2-1 / \alpha} /\left(\alpha \sqrt{N_{E}}\right)$ & $(1-p)^{-1 / \alpha}$ \\
Exponential & $p^{1 / 2}(1-p)^{-1 / 2} /\left(\sqrt{N_{E}}\right)$ & $-\log (1-p)$ \\
\hline
\end{tabular}

$c_{2}(p)=\frac{1}{\sqrt{q}} \quad f_{2}(r)=\sqrt{\frac{1+r}{(1-r)^{3}}}$

For the Pareto distribution we have fitted numerical results for $C V$ and mean firing time shown in Fig. 6 and Fig. $5 b$ by

$C V=\sqrt{\frac{1+r}{N_{\mathrm{th}}(1-r)^{2.3}}} \quad E T=\frac{q}{(1-r)^{1.65}}$

from which output jitter can be obtained

$\sigma_{\text {out }}=C V \cdot E T$

which fits well with the numerical results as shown in Fig. $1 b$.

\section{Discussion}

There has been much recent research on the I\&F model to clarify a few important aspects of neural computation. In the present paper we provide a systematic approach to the relationship between input and output, in particular, concentrating on the effects of variation in the time domain and on the balance between excitation and inhibition of the input. Some results which have not been elucidated in the literature are presented here to stimulate further research. For a given short-tailed input distribution, in the range of values of $r$ in which the model produces a physiological $C V$, output jitter is in general larger than input jitter. Only when the $C V$ is smaller than 0.5 or the input distribution is a long-tailed one will output jitter converge, the phenomenon observed in [21]. Keeping the ratio between inhibitory inputs and excitatory inputs constant, the longer the tail, the smaller the output jitter divided by the input jitter. Output jitter is an increasing function of the ratio between the numbers of inhibitory and excitatory synapses and a decreasing function of the length of the input distribution tail. The I\&F model tends to enlarge small input jitter and reduce large input jitter. With equal excitatory and inhibitory inputs as considered in [24], an output distribution with a long tail will result. ISI $C V$ is an increasing function of both tail length and the ratio, $r$. The longer the tail, the lesser is the requirement of balance between IPSPs and EPSPs for the model to produce a physiologically reasonable $C V$. Les us consider some further aspects.

Network or hierarchy behaviour [14]. In this paper we only consider the input-output relationship of a single neuron. A more interesting question is that, if the output of an I\&F model is an input of another, or another sequence of I\&F models, what will be the behaviour of the final output. It is generally believed that real neurones with dendritic trees form a hierarchy of I\&F models. Hence even to understand a single neuron's behaviour, it seems necessary for us to consider the output of the model as the input of another model. As is revealed in the current paper, a short-tailed input, as a result of the balance between excitatory and inhibitory 
inputs, can result in a long-tailed output, even for the Poisson case. We expect a rich range of behaviour will be observed for the output of networks of I\&F models.

Leakage. We have not included leakage in the model presented here for the following two reasons: we wish to have a convenient comparison with results presented in $[21,24,25,28]$, and we have analytical results available only for the case of no leakage. What will be the influence of leakage on our results? In a sense we could think of leakage as a further inhibitory input, and therefore it might help us to reduce the requirement for balance between inhibitory and excitatory inputs. When the leakage is small, our results certainly still hold true. The I\&F model with leakage is called Stein's model and has been intensively studied in the literature for Poisson inputs, mainly numerically. In particular, if we approximate Stein's model by a diffusion model - a traditional route taken to approximate random walks - we are able to obtain some analytical results related to $T$, the first exit time for the diffusion process [11, 22, 23]. There are also corresponding approximations for long tail distributed random walks. The derived Brownian is called fractional Brownian motion [3]. In the future, we hope it will be possible to develop analogous results for the case of a short tail random walk or its approximation, standard Brownian motion.

Acknowledgements. We are grateful to Waibun Fu, Gareth Leng, Brunello Tirozzi and an anonymous referee for their valuable comments on an earlier version of this article. Figure $4 \mathrm{a}$ was prepared by Waibun Fu. The work was financially supported by the BBSRC and an ESEP of the Royal Society.

\section{References}

1. Abbott LF, Varela JA, Sen K, Nelson SB (1997) Synaptic depression and cortical gain control. Science 275:220-223

2. Abeles M (1990) Corticonics. Cambridge University Press, Cambridge, UK

3. Albeverio S Fenstad JE, Heøgh-Krohn, Lindstrøm T (1986) Nonstandard methods in stochastic analysis and mathematical physics. Academic Press, New York

4. Balkema AA, De Haan L (1978a) Limit distributions for order statistics. I. Theory of probability and its applications, XXIII, 77-92

5. Balkema AA, De Haan L (1978b) Limit distributions for order statistics. II. Theory of probability and its applications, XXIII, 341-358

6. Bugmann G, Christodoulou C, Taylor JG (1997) Role of temporal integration and fluctuation detection in the highly irregular firing of a leaky integrator neuron models with partial reset. Neural Comput 9:985-1000

7. Feller W (1966) An introduction to probability theory and its applications. John Wiley, New York
8. Feng $\mathbf{J}$ (1996) The hydrodynamic limit for the reaction diffusion equation - an approach in terms of the GPV method. $\mathbf{J}$ Theor Probab 9:285-299

9. Feng J (1997) Behaviours of spike output jitter in the integrateand-fire model. Phys Rev Lett 79:4505-4508

10. Feng J, Brown D (1998) Spike output jitter, mean firing time and coefficient of variation. J Phys A Math Gen, 31:1239-1252

11. Feng J, Lei G, Qian M (1992) Second-order algorithms for SDE. J Comput Math 10:376-387

12. Gerstein GL, Mandelbrot B (1964) Random walk models for the spike activity of a single neuron. Biophys $\mathrm{J} 4: 41-68$

13. Holden AV (1976) Models of the stochastic activity of neurones. Springer, Berlin, Heidelberg, New York

14. Hopfield JJ, Herz AVM (1995) Rapid local synchronization of action potentials: towards computation with coupled integrateand-fire networks. Proc Natl Acad Sci USA 92:6655-6662

15. Inoue J, Sato S, Ricciardi LM (1995) On the parameter-estimation for diffusion-models of single neurons activities. 1. Application to spontaneuos activities of mesencephalic reticular-formation cells in sleep and waking states. Biol Cybern 73:209-221

16. Lánský P, Radil T (1987) Statisitical-inference on spontaneous neuronal discharge patterns. 1. Single neuron. Biol Cybern 55:299-311

17. Lánský P, Smith CE (1989) The effect of a random initial-value in neural first-passage-time models. Math Biosci 93:191-215

18. Knight B (1972) Dynamics of encoding in a population of neurones. J Gen Physiol 59:734-766

19. Koch C (1997) Computation and the single neuron. Nature, 385:207-210

20. Leadbetter MR, Lindgren G, Rootzén H (1983) Extremes and related properties of random sequences and processes. Springer, Berlin, Heidelberg, New York

21. Marsalek P, Koch C, Maunsell J (1997) On the relationship between synaptic input and spike output jitter in individual neurones. Proc Nat Acad Sci USA 94:735-740

22. Musila M, Lánský P (1994) On the interspike intervals calculated from diffusion approximations for Stein's neuronal model with reversal potentials. J Theor Biol 171:225-232

23. Ricciardi LM, Sato S (1990) Diffusion process and first-passage-times problems. In: Ricciardi LM (ed) Lectures in applied mathematics and informatics. Manchester University Press, Manchester

24. Shadlen MN, Newsome WT (1994) Noise, neural codes and cortical organization. Curr Opin Neurobiol 4:569-579

25. Softky W, Koch C (1993) The highly irregular firing of corticalcells is inconsistent with temporal integration of random EPSPs. J Neurosci 13:334-350

26. Terman D, Bose A, Kopell N (1996) Functional reorganization in thalamocortical networks: transition between spindling and delta sleep rhythms. Proc Nat Acad Sci USA 93:15417-15422

27. Thomson AM (1997) Activity-dependent properties of synaptic transmission at two classes of connections made by rat neocortical pyramidal. J Physiol 502:131-147

28. Troyer TW, Miller KD (1997) Physiological gain leads to high ISI variability in a simple model of a cortical regular spiking cell. Neural Comput 9:733-745

29. Tuckwell HC (1988) Introduction to theoretical neurobiology Vol 2. Cambridge University Press, Cambridge, UK 